\title{
Coexistence of Perineural Invasion and Lymph Node Metastases Is a Poor Prognostic Factor in Patients with Locally Advanced Rectal Cancer after Preoperative Chemoradiotherapy Followed by Radical Resection and Adjuvant Chemotherapy
}

\author{
Chun-Ming Huang ${ }^{a, e} \quad$ Ching-Wen Huang ${ }^{b, e, h}$ Ming-Yii Huang ${ }^{a, j}$ \\ Chih-Hung Lin ${ }^{c}$ Chin-Fan Chen ${ }^{i}$ Yung-Sung Yeh ${ }^{f, i}$ Cheng-Jen Ma ${ }^{b}$ \\ Chih-Jen Huang ${ }^{a, j}$ Jaw-Yuan Wang ${ }^{b, d, f, g, k}$
}

\begin{abstract}
Departments of a Radiation Oncology, ${ }^{b}$ Surgery, ${ }^{c}$ Pathology and ${ }^{d}$ Cancer Center, Kaohsiung Medical University Hospital, Kaohsiung Medical University, ${ }^{e}$ Graduate Institute of Medicine, ${ }^{f}$ Graduate Institute of Clinical Medicine, g Department of Medical Genetics, College of Medicine, Kaohsiung Medical University, Departments of h Surgery and 'Emergency Medicine, Kaohsiung Municipal Hsiao-Kang Hospital, and Departments of ${ }^{j}$ Radiation Oncology and kSurgery, Faculty of Medicine, College of Medicine, Kaohsiung Medical University, Kaohsiung, Taiwan, ROC
\end{abstract}

\section{Key Words}

Perineural invasion - Lymph node metastases ·

Rectal cancer · Chemoradiotherapy

\begin{abstract}
Objective: To determine the role of lymph node metastases (ypN) and perineural invasion (PNI) in patients with locally advanced rectal cancer (LARC). Subjects and Methods: Eighty-eight LARC patients receiving preoperative chemoradiotherapy from April 2006 to November 2011 were enrolled in this study. Univariate and multivariate analyses were conducted to determine the association between clinicopathologic features and clinical outcome. Results: The presence of ypN $(p=0.011)$ and PNI $(p=0.032)$ was a significant adverse
\end{abstract}

\section{KARGER}

E-Mail karger@karger.com www.karger.com/mpp
(C) 2014 S. Karger AG, Basel 1011-7571/14/0235-0465\$39.50/0 Open access

This is an Open Access article licensed under the terms of the Creative Commons Attribution-NonCommercial 3.0 Unported license (CC BY-NC) (www.karger.com/OA-license), applicable to the online version of the article only. Distribution permitted for non-commercial purposes only. prognostic factor for disease-free survival (DFS). High histologic grade $(p=0.015), P N I+(p=0.043)$ and ypN+ $(p=0.041)$ were adverse prognostic factors for overall survival (OS). Positive PNI was significantly associated with a higher risk of distant failure (odds ratio $=6.09 ; 95 \% \mathrm{Cl}: 1.57-27.05 ; \mathrm{p}=0.008$ ). Moreover, patients with a coexistence of $y p N+$ and $\mathrm{PNI}+$ had the significantly worst DFS $(p<0.001)$ and OS rates ( $<$ 0.001 ) compared with other phenotypes. Conclusions: The presence of either PNI or ypN was a significant prognostic factor for predicting poor survival rates in LARC patients, especially those with a coexistence of both factors. Accordingly, we recommend an intensive follow-up and therapeutic programs for LARC patients with simultaneous $\mathrm{PNI}+$ and ypN+.

(c) 2014 S. Karger AG, Basel
Prof. Jaw-Yuan Wang, MD, $\mathrm{PhD}$

Division of Gastrointestinal and General Surgery, Department of Surgery Kaohsiung Medical University Hospital, Kaohsiung Medical University No. 100 Tzyou 1st Road, Kaohsiung 807, Taiwan (ROC)

E-Mail cy614112@ms14.hinet.net 


\section{Introduction}

Preoperative chemoradiotherapy (CRT) has improved the locoregional control rate but not the overall survival (OS) rate in patients with locally advanced rectal cancer (LARC) compared with postoperative CRT $[1,2]$. Distant recurrence is at present the leading cause of treatment failure in the preoperative CRT era, and distant failure rates range from 30 to $40 \%$ [1]. However, the benefit of postoperative adjuvant chemotherapy in LARC patients undergoing preoperative CRT remains controversial [36]. Therefore, it is crucial to identify such patients at high risk of distant recurrence after multimodality therapy. The high-risk patients theoretically benefit from adjuvant chemotherapy.

The most significant prognostic factors in patients with LARC are lymph node metastases [7-9]. Patients with lymph node metastases have significantly worse disease-free (DFS) and OS rates compared with those without lymph node metastases. For rectal cancer, several reports have suggested the presence of perineural invasion (PNI) as an independent prognostic factor for predicting survival time $[7,10]$.

No relevant studies have investigated the correlation between ypN and PNI status, and the effect of their coexistence on the oncologic outcome in LARC patients after multimodality therapy. Hence, the purpose of this study was to evaluate the impact of the coexistence of $\mathrm{ypN}+$ and $\mathrm{PNI}+$ on the clinical outcome in LARC patients undergoing preoperative CRT followed by radical resection and adjuvant chemotherapy.

\section{Subjects and Methods}

\section{Patients and Evaluation}

This was a retrospective study that reviewed LARC patients treated with preoperative CRT followed by radical resection and adjuvant chemotherapy. The inclusion criteria for the study were as follows: (1) biopsy-proven rectal adenocarcinoma; (2) locally advanced diseases (clinical T3-4 or nodal involvement); (3) tumors located within $10 \mathrm{~cm}$ of the anal verge; (4) Eastern Cooperative Oncology Group performance status $\leq 2$, and (5) no evidence of distant metastasis. Exclusion criteria included local excision of tumor, radiation dose $<45 \mathrm{~Gy}$, a positive surgical margin, history of earlier pelvic irradiation, and history of malignancies other than rectal cancer. The medical records of 103 patients from April 2006 to November 2011 were reviewed, and the Institutional Review Board approved the study.

\section{Treatment}

All patients had received preoperative CRT followed by radical resection and adjuvant chemotherapy. The details of the treatment
Table 1. Clinicopathologic characteristics of 88 patients

\begin{tabular}{|c|c|}
\hline Median age (range), years & $64.5(34-85)$ \\
\hline \multicolumn{2}{|l|}{ Gender } \\
\hline Males & $50(56.8)$ \\
\hline Females & $38(43.2)$ \\
\hline \multicolumn{2}{|l|}{ Pre-CRT CEA, ng/ml } \\
\hline$<5$ & $55(62.5)$ \\
\hline$\geq 5$ & $33(37.5)$ \\
\hline \multicolumn{2}{|l|}{ Clinical tumor stage } \\
\hline cT1-2 & $8(9.1)$ \\
\hline cT3 & $72(81.8)$ \\
\hline cT4 & $8(9.1)$ \\
\hline \multicolumn{2}{|l|}{ Clinical nodal stage } \\
\hline cN0 & $19(21.6)$ \\
\hline $\mathrm{cN} 1$ & $41(46.6)$ \\
\hline $\mathrm{cN} 2$ & $28(31.8)$ \\
\hline \multicolumn{2}{|c|}{ Distance of tumor from anal verge, $\mathrm{cm}$} \\
\hline$<5$ & $47(53.4)$ \\
\hline $5-10$ & $41(46.6)$ \\
\hline \multicolumn{2}{|l|}{ Pathologic T category } \\
\hline урТ0 & $15(17.1)$ \\
\hline ypT1 & $3(3.5)$ \\
\hline урТ2 & $27(30.6)$ \\
\hline урТ3 & $38(43.2)$ \\
\hline урТ4 & $5(5.6)$ \\
\hline \multicolumn{2}{|l|}{ Pathologic $N$ category } \\
\hline ypNo & $65(73.8)$ \\
\hline ypN1 & $19(21.6)$ \\
\hline ypN2 & $4(4.6)$ \\
\hline \multicolumn{2}{|l|}{ Tumor grade ${ }^{\mathrm{a}}$} \\
\hline Low & $82(93.2)$ \\
\hline High & $6(6.8)$ \\
\hline \multicolumn{2}{|l|}{ PNI } \\
\hline Negative & $64(72.7)$ \\
\hline Positive & $24(27.3)$ \\
\hline \multicolumn{2}{|l|}{ Lymphovascular invasion } \\
\hline Negative & $80(90.9)$ \\
\hline Positive & $8(9.1)$ \\
\hline
\end{tabular}

Unless otherwise indicated values represent number with the percentage in parentheses. CEA = Carcinoembryonic antigen. ${ }^{\text {a }}$ Low grade represents well or moderately differentiated histology and high grade represents poorly differentiated histology or mucinous carcinoma.

course were reported previously [11]. In brief, radiotherapy (RT) was administered to the whole pelvis at a dose of $45 \mathrm{~Gy}$ in $25 \mathrm{frac}-$ tions with or without a boost of 5.4 Gy in 3 fractions to the primary tumor. Of the 88 patients, 42 patients were treated with 5-fluorouracil $\left(350 \mathrm{mg} / \mathrm{m}^{2}\right.$ intravenous bolus) and leucovorin $\left(20 \mathrm{mg} / \mathrm{m}^{2}\right.$ intravenous bolus) during RT. Forty-six patients received capecitabine ( $850 \mathrm{mg} / \mathrm{m}^{2}$, twice daily, 5 days per week).

Patients underwent total mesorectal excision 6-10 weeks after the completion of CRT. Four to 6 weeks after radical surgery, all patients received the same adjuvant chemotherapy regimens as before surgery. 
Table 2. Univariate and multivariate analyses for pathologic factors/recurrence associated with PNI

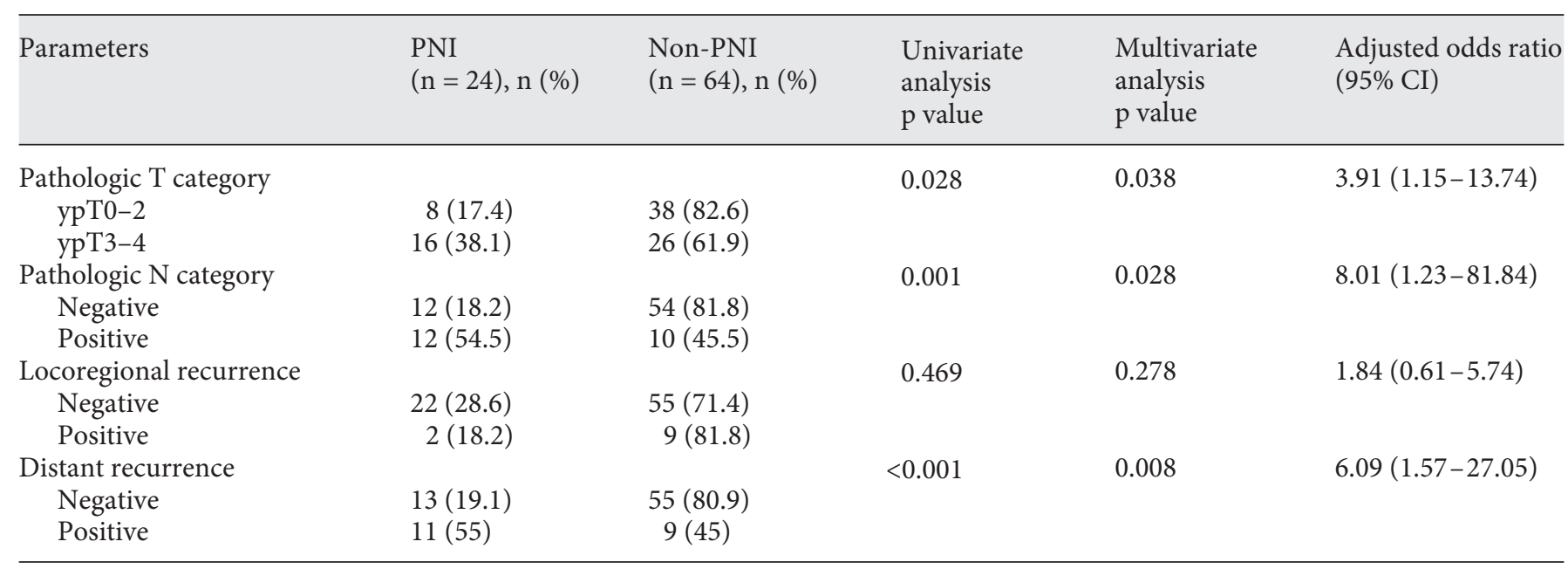

yp: the ' $y$ ' prefix added to the pathologic (p) stage designates a TNM stage that is assigned after multimodality therapy. CI = Confidence interval.

\section{Pathology Review}

Two experienced pathologists (C.-H.L. and C.-Y.C.) analyzed all resected specimens using the standard method. Cases experiencing pathologic complete response were categorized as lymphovascular invasion and PNI-. PNI was defined as tumor cells within any layer of the nerve sheath or tumor in the perineural space that involved at least one third of the nerve circumference [13]. Patients with positive PNI were further subclassified into intramural PNI (neural invasion was seen only in the proper muscle layer) and extramural PNI (neural invasion was seen beyond the proper muscle layer) [12]. For the prognostic evaluation of PNI+ and ypN+, 12 patients were categorized as the $\mathrm{PNI}+/ \mathrm{ypN}+$ group and another 76 as having other phenotypes.

\section{Statistical Analysis}

$\chi^{2}$ and logistic regression model were performed to identify associations between potential variables and PNI. DFS was defined as the time from starting RT to any type of recurrence of disease. OS was defined as the time from the start of RT to death from any cause. Survival analyses were estimated by the Kaplan-Meier method and compared by the log-rank test. A Cox proportional hazards model with forward stepwise variable selection was performed for survival analysis. Data analyses were performed with the JMP software (version 9.0, SAS Institute Inc., Cary, N.C., USA). Results were considered significant at $\mathrm{p}<0.05$.

\section{Results}

\section{Patient Demographics}

Of the 15 patients who were excluded, 4 had received local excision, 9 had evidence of distant metastases, and 2 had double cancer. The clinicopathologic characteristics of the 88 patients analyzed are listed in table 1 . The mean follow-up was 31 months, and the median was 27 months.

\section{Parameters Affect OS and DFS}

Of the 88 patients, $22(25 \%)$ had a relapse and $14(15.9 \%)$ died. The overall 5-year DFS rate was 57.6\%, and the 5-year OS rate was $66.9 \%$. On univariate analysis, the ypT category, the ypN category, the pCR status, the histologic grade, and PNI were significantly associated with DFS. Using a multivariate model, $y \mathrm{pN}+(\mathrm{p}=0.011)$ and $\mathrm{PNI}+(\mathrm{p}=$ 0.032 ) were significantly adverse factors that affected DFS. On univariate analysis, the ypN category, the histologic grade, lymphovascular invasion, and PNI significantly affected the OS. On multivariate analysis, $y p N+(p=0.041)$, high-grade histology $(\mathrm{p}=0.015)$, and PNI+ $(\mathrm{p}=0.043)$ were significantly adverse factors for the OS.

\section{Association between PNI and Pathologic Stage and Recurrence}

Of the 88 patients, $24(27.3 \%)$ had a positive PNI. The association between PNI and ypT, ypN and recurrence is shown in table 2. Multivariate analysis confirmed that the $y p T$ category $(\mathrm{p}=0.038)$, the $y \mathrm{pN}$ category $(\mathrm{p}=0.028)$, and distant recurrence $(\mathrm{p}=0.008)$ were significant factors associated with the PNI status in the LARC patients. The 5 -year DFS rate was significantly higher in the PNIgroup than the PNI+ group (61.1 vs. $50.7 \%, \mathrm{p}=0.025)$. Patients with PNI- tumors had a higher 5-year OS rate compared with patients with PNI+ tumors (80.1 vs. $37.4 \%, \mathrm{p}=0.001)$. Of 24 patients with PNI+ tumors, 10 


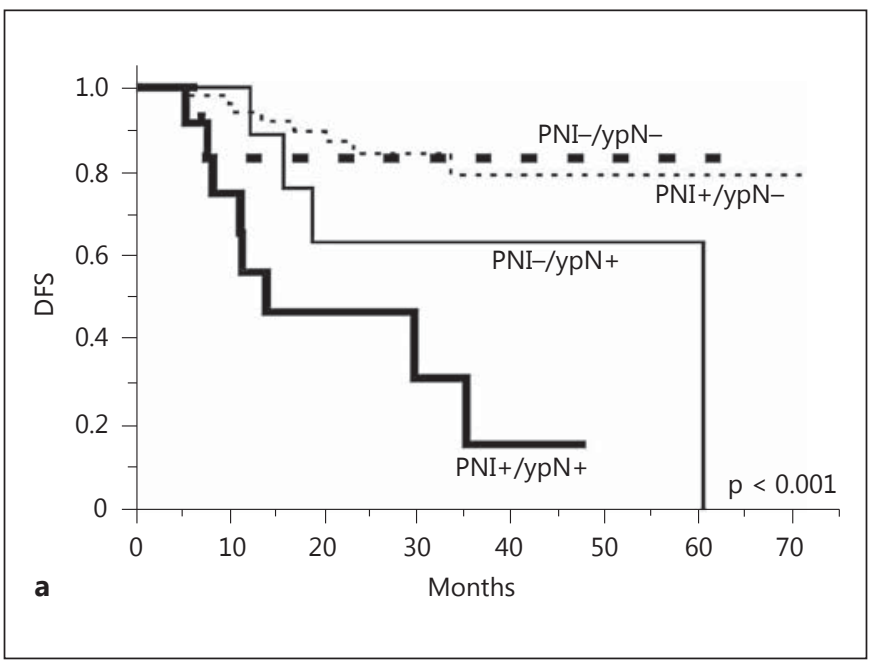

Fig. 1. Cumulative survival rates of the 88 patients with LARC undergoing preoperative CRT and radical resection. a The DFS in LARC patients with a coexistence of ypN and PNI was significantly lower than that in LARC patients with other phenotypes $(\mathrm{p}<$

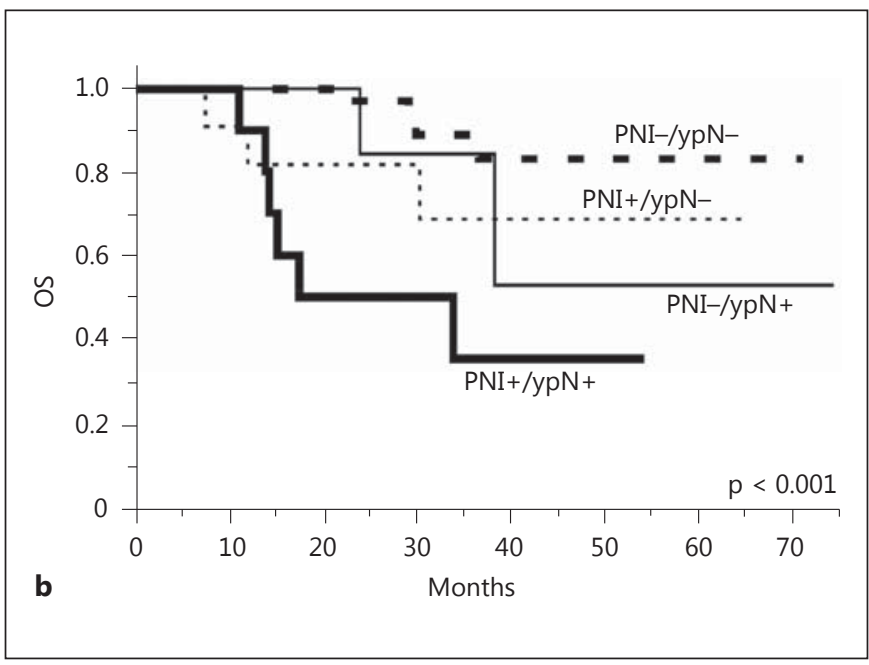

0.001). b The OS in LARC patients with a coexistence of ypN and PNI was lower than that in LARC patients with other phenotypes $(\mathrm{p}<0.001)$.

Table 3. Combination of PNI and lymph node metastases as predictors of a decreased survival for patients with LARC

\begin{tabular}{|c|c|c|c|c|c|c|c|}
\hline & $\begin{array}{l}\text { Patients, } \\
\mathrm{n}(\%)\end{array}$ & \multicolumn{3}{|l|}{ DFS } & \multicolumn{3}{|l|}{ OS } \\
\hline Any predictor & & 0.009 & $2.72(1.06-7.23)$ & 0.065 & 0.002 & $3.96(1.12-15.12)$ & 0.008 \\
\hline Positive & $35(40)$ & & & & & & \\
\hline Negative & $53(60)$ & & & & & & \\
\hline Negative & $76(86)$ & & & & & & \\
\hline
\end{tabular}

$\mathrm{HR}=$ Hazard ratio; $\mathrm{CI}=$ confidence interval.

(41.67\%) had extramural PNI and 14 had intramural PNI. The 5-year DFS for patients with PNI- tumors, intramural PNI, and extramural PNI were 61.1, 70.2, and $0 \%$, respectively $(\mathrm{p}<0.001)$. The 5 -year OS rates for patients with negative PNI, intramural PNI, and extramural PNI were $80.1,75.6$, and $0 \%$, respectively $(\mathrm{p}<0.001)$.

\section{Correlation between PNI+/ypN+ and Clinical}

Outcome

The DFS in LARC patients with PNI+/ypN+ was significantly lower than that in LARC patients with other phenotypes (fig. 1a). Furthermore, LARC patients with PNI+/ $y p N+$ had a significantly shorter OS time than that in
LARC patients with other phenotypes (fig. 1b). The results of combining ypN and PNI as predictors of decreased survival time are shown in table 3. Notably, the coexistence of $\mathrm{ypN}$ and PNI in the resected tumors exhibited a 5-fold increase in the likelihood of developing LARC recurrence and a 7-fold increase of dying in LARC in our patients.

\section{Discussion}

In this study, we found that $\mathrm{ypN}$ and PNI were independent factors for predicting survival in LARC patients undergoing preoperative CRT followed by radical resection 
and adjuvant chemotherapy. We also demonstrated that LARC patients with positive PNI tended to develop distant recurrence. Moreover, patients with a coexistence of ypN+ and PNI+ had the significantly worst DFS and OS rates.

According to our data, positive PNI was an adverse prognostic factor in patients with rectal cancer after preoperative CRT and correlated with poor OS and DFS. Moreover, patients with positive PNI were more likely to have distant recurrence than those without PNI. The prognostic significance of PNI positivity in LARC patients after multimodality therapy has been debated. Ceyhan et al. [10] reported that the severity of neural invasion was significantly associated with poor survival and an increased local recurrence rate in rectal cancer patients after preoperative CRT. Guillem et al. [9] demonstrated that the presence of PNI was associated with decreased OS and DFS at 10 years. In contrast, several reports have shown that PNI was not significantly associated with the oncologic outcome in LARC patients undergoing multimodality therapy $[8,15]$. One of the possible explanations of this controversy might be the depth of neural invasion. In this study, the LARC patients with intramural PNI had a similar survival rate to those with negative PNI, but the survival rate of patients with extramural PNI was inferior. Our findings were comparable to what Ueno el al. [12] reported. Since the depth of neural invasion may influence clinical outcomes, this may be of importance when discussing conflicting data on the prognostic impact.

In our series, patients with PNI+ or ypN+ tumors had dismal survival and disease control rates despite adjuvant chemotherapy after radical resection. All patients received adjuvant chemotherapy and $22(25 \%)$ of 88 experienced recurrence. In addition, the pattern of recurrence was mostly distant failure. However, patients without adverse pathologic features also received adjuvant chemotherapy, and they had superior survival. The benefit and necessity of adjuvant chemotherapy for LARC patients after preoperative CRT have been under debate, although according to the NCCN Guidelines adjuvant chemotherapy should be given to LARC patients even if there is tumor response to CRT [16]. Several studies have reported superior survival for LARC patients receiving fluoropyrimidine-based adjuvant chemotherapy $[6,8]$, but some have shown no survival benefit $[3,4]$. Collette et al. [17] showed that patients with ypT0-2 after CRT benefited from adjuvant chemotherapy in terms of better DFS and OS than those with ypT3-4. However, overtreatment is a big concern when administering chemotherapy postoperatively to relatively low-risk patients (ypT0-2 vs. ypT34). Pathologic parameters are good indicators to stratify

Perineural Invasion and Lymph Node Metastases in Rectal Cancer patients' prognosis and to guide adjuvant chemotherapy $[18,19]$. In this study, we demonstrated that the PNI and ypN status could stratify the prognosis of LARC patients after multimodality treatment. The PNI- and the ypNgroup, which was shown to be associated with a better prognosis after preoperative CRT, might be better treated without additional adjuvant therapy. Conversely, patients with PNI+ and ypN+ may be preferable candidates for more potent chemotherapy agents because these groups have the worst survival anticipated by currently used adjuvant 5-fluorouracil. Additional future studies are needed to verify these findings.

Two definitions of PNI were generally used in the literature: tumor cells invading the perineural space that involved at least one third of the nerve (surround-sheath PNI) or tumor within any of the three layers (epineurium, perineurium, or endoneurium) of the nerve sheath (through-sheath PNI) [13]. Peng et al. [14] evaluated the effect of PNI on 173 patients with pT3N0 rectal cancer, and both the surround-sheath PNI and through-sheath PNI groups had a similar 5-year local recurrence rate, which was significantly higher than that of the PNIgroup. As a result, we viewed both as definitions of positive PNI in this study. The incidence of PNI in rectal cancer patients ranges between 14 and 30\% [13, 14]. In our study, $27.3 \%$ (24 of 88 patients) had PNI, and this is consistent with other reports.

This retrospective study had certain limitations regardless of the significant correlation between PNI status and survival. First, the sample size was relatively small, and this may minimize the detection of small but clinically important parameters. Second, interobserver or intraobserver variability of PNI status may exist. As described above, the dispute about the definition of PNI will lead to a variable PNI status among pathologists. Furthermore, inflammation or large mucinous pools may make it difficult to detect the presence of tumor around nerves.

\section{Conclusions}

In addition to the ypN status, PNI was another independent prognostic factor for predicting survival rates in LARC patients after preoperative CRT radical resection and adjuvant chemotherapy. Moreover, LARC patients with a coexistence of $\mathrm{ypN}$ and PNI had the worst outcome. These patients would benefit from intensive follow-up and therapeutic programs. However, a largescale, prospective randomized study is needed to confirm our findings. 


\section{Acknowledgements}

The authors acknowledge the contribution to the collection of data made by the Colorectal Cancer Group from the Cancer Center of Kaohsiung Medical University Hospital. This study was supported by grants from the Excellence for Cancer Research Center
Grant through the funding by the Ministry of Health and Welfare, Executive Yuan, Taiwan, ROC (MOHW103-TD-B-111-05), the Kaohsiung Medical University Hospital (KMUH100-0M49), and the Grant of Biosignature in Colorectal Cancers, Academia Sinica, Taiwan, ROC.

\section{References}

1 Sauer R, Becker H, Hohenberger W, et al: Preoperative versus postoperative chemoradiotherapy for rectal cancer. N Engl J Med 2004; 351:1731-1740

-2 Roh MS, Colangelo LH, O'Connell MJ, et al: Preoperative multimodality therapy improves disease-free survival in patients with carcinoma of the rectum: NSABP R-03. J Clin Oncol 2009;27:5124-5130.

-3 Fietkau R, Barten M, Klautke G, et al: Postoperative chemotherapy may not be necessary for patients with ypN0-category after neoadjuvant chemoradiotherapy of rectal cancer. Dis Colon Rectum 2006;49:1284-1292.

4 Kiran RP, Kirat HT, Burgess AN, et al: Is adjuvant chemotherapy really needed after curative surgery for rectal cancer patients who are node-negative after neoadjuvant chemoradiotherapy? Ann Surg Oncol 2012;19: 1206-1212.

5 Bosset JF, Collette L, Calais G, et al: Chemotherapy with preoperative radiotherapy in rectal cancer. N Engl J Med 2006;355:11141123.

6 Janjan NA, Crane C, Feig BW, et al: Improved overall survival among responders to preoperative chemoradiation for locally advanced rectal cancer. Am J Clin Oncol 2001;24:107112.
7 Kim NK, Kim YW, Min BS, et al: Factors associated with local recurrence after neoadjuvant chemoradiation with total mesorectal excision for rectal cancer. World J Surg 2009; 33:1741-1749.

8 Das P, Skibber JM, Rodriguez-Bigas MA, et al: Clinical and pathologic predictors of locoregional recurrence, distant metastasis, and overall survival in patients treated with chemoradiation and mesorectal excision for rectal cancer. Am J Clin Oncol 2006;29:219224.

-9 Guillem JG, Chessin DB, Cohen AM, et al: Long-term oncologic outcome following preoperative combined modality therapy and total mesorectal excision of locally advanced rectal cancer. Ann Surg 2005;241:829-838.

10 Ceyhan GO, Liebl F, Maak M, et al: The severity of neural invasion is a crucial prognostic factor in rectal cancer independent of neoadjuvant radiochemotherapy. Ann Surg 2010; 252:797-804.

11 Chen CF, Huang MY, Huang CJ, et al: A observational study of the efficacy and safety of capecitabine versus bolus infusional 5-fluorouracil in pre-operative chemoradiotherapy for locally advanced rectal cancer. Int J Colorectal Dis 2012;27:727-736.

12 Ueno H, Mochizuki H, Hase K, et al: A study on prognostic value of neural invasion in patients with rectal cancer. Jpn J Gastroenterol Surg 1994;27:2126-2134.

13 Liebig C, Ayala G, Wilks J, et al: Perineural invasion is an independent predictor of outcome in colorectal cancer. J Clin Oncol 2009; 27:5131-5137.
14 Peng J, Sheng W, Huang D, et al: Perineural invasion in pT3N0 rectal cancer: the incidence and its prognostic effect. Cancer 2011; 117:1415-1421.

15 Lim SB, Yu CS, Hong YS, et al: Long-term outcomes in patients with locally advanced rectal cancer treated with preoperative chemoradiation followed by curative surgical resection. J Surg Oncol 2012;106:659-666.

16 Khrizman P, Niland JC, ter Veer A, et al: Postoperative adjuvant chemotherapy use in patients with stage II/III rectal cancer treated with neoadjuvant therapy: a national comprehensive cancer network analysis. J Clin Oncol 2013;31:30-38.

17 Collette L, Bosset JF, den Dulk M, et al: Patients with curative resection of cT3-4 rectal cancer after preoperative radiotherapy or radiochemotherapy: does anybody benefit from adjuvant fluorouracil-based chemotherapy? J Clin Oncol 2007;25:4379-4386.

18 Michael JD: Tumor markers in clinical practice: a review focusing on common solid cancers. Med Princ Pract 2013;22:4-11.

19 Fujita S, Shimoda T, Yoshimura K, et al: Prospective evaluation of prognostic factors in patients with colorectal cancer undergoing curative resection. J Surg Oncol 2003;84:127131 